\title{
Agent Solutions for E-business Transactions
}

\author{
Ismail Khalil Ibrahim, Wieland Schwinger, Edgar Weippl, Josef Altmann, Werner Winiwarter \\ Software Competence Center Hagenberg, Hauptstrasse 99, A-4232 Hagenberg, Austria \\ \{ismail.khalil-ibrahim,wieland.schwinger, edgar.weippl, josef.altmann,werner.winiwarter\}@scch.at
}

\begin{abstract}
Software agents have become very popular in the last few years. They have been used successfully to perform quite a diverse range of applications. In this paper we present a model for agents as mediators in e-commerce, which highlights how far technology can be used to automate e-business transactions.
\end{abstract}

\section{Introduction}

E-commerce [9] is the ability to perform business transactions involving the exchange of information, goods, services, and payments between two or more parties using electronic tools and technologies.

The personalized, continuously running, and semiautonomous properties of software agents make them wellsuited for e-business applications [5] representing the interests of customers, suppliers, or intermediaries [6]. Unfortunately, conventional e-commerce models [8] can not be adapted for studying the roles of agents as mediators in e-commerce because these models are built upon functional components, such as marketing, shopping, ordering, fulfillment, and customer services, and for each functional component different applications, infrastructures, and systems are put in place. Instead it is useful to study the roles of agents as mediators in e-commerce in the context of a common model that cuts across all disciplines involved in e-commerce [4] such as marketing, computer science, consumer behavior, management, business law, and ethics. Such a model provides tools necessary to understand the relationships and interactions between different functional components.

In this paper, we present a model for agent-based ebusiness transactions, which covers all dimensions of ebusiness with respect to the main issues enabling companies to conduct e-business transactions.

The paper is organized as follows. Section 2 presents our three-dimensional model for investigating the roles of agents in mediating e-commerce transactions. Section 3 gives a brief overview of the roles that agents can perform in the integral value added chain of e-commerce. In Section 4 we highlight key issues in the design of such agents. We conclude in Section 5 with related and future work.

\section{A Generic Model for Agents as Mediators in Electronic Commerce}

In this section, we present a model for studying which roles agents can perform for mediating e-commerce transactions. The model comprises three main dimensions of e-commerce (see Fig. 1), i.e. the technical, business, and organizational dimension [2]. For each dimension, we explore the main issues that are necessary to understand the relations and interactions among different components of ecommerce. Furthermore, this will set up the environment to identify where agents can play important roles in the integral value added chain of e-commerce.

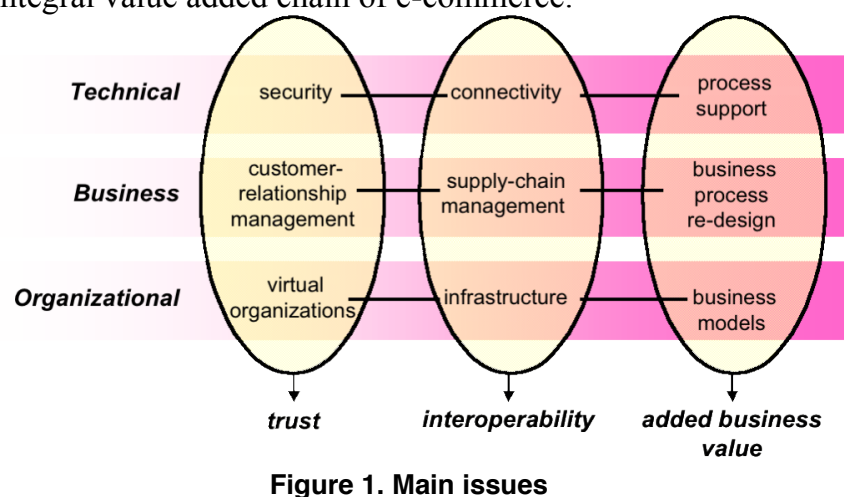

\subsection{Dimensions: Technical, Business, and Organizational Issues}

There are several technical issues that require attention in e-commerce [4]. The most noticeable one is security. The second is the interoperability of the systems of the parties involved in the e-commerce transaction. The third is the support of business processes and trade procedures.

The business dimension of e-commerce [9] is also broad. In order to allow businesses to benefit from emerging technologies, business processes and business models are subject to redesign including business process reengineering, customer relationship management, and supply chain management.

In the core of the organizational dimension [6] are the emergence of virtual organizations, the new business models, and the infrastructures necessary to facilitate ecommerce transactions. 
The dimensions along with the related main issues are shown in Fig. 1. It is clear that these issues are closely related to each other.

\subsection{Context: Added Business Value, Interoperability, and Trustworthiness}

With regard to the roles of software agents in the integral value added chain of e-commerce, focus should be on three central issues: added business value, interoperability, and trustworthiness [2].

Added business value: The potential of e-commerce lies in its transformational power. Markets with their economical, social, and technological factors are creating a highly competitive environment for which companies need to redefine how they conduct business, which business strategies to adopt, and which operational and organizational areas to change [9]. This implies the need to redesign business processes, to change consumer-supplier relationships, and above all to make companies and consumers to accept e-commerce.

To achieve this, companies planning to use e-commerce have to provide a real added value to their business partners and customers. This necessitates the need for new technologies and for a regulatory framework to support of e-commerce transactions [4].

Interoperability: Interoperability is the ability of distinct, pre-existing, independent systems to work together in achieving a common goal [3]. Although interoperability is predominantly a technical issue, it has major implications for the business and organizational elements of the ecommerce world. For organizations, interoperability facilitates the coupling of business processes in different organizations [2]. This implies that the organizational framework for transactions across borders should be interoperable as well.

Trustworthiness: Trust is an important issue in ecommerce. In electronic environments, classic trust existing between parties knowing each other personally is partly replaced by electronic trust based on technologies, such as encryption and the use of trusted third parties [1]. However, such new technologies for building trust do not fit seamlessly into the organizational system.

\subsection{Relationships: E-markets, Business Models, and Actors}

Electronic markets: Markets (electronic or otherwise) have three main functions [7]: i) matching buyers and sellers; ii) facilitating the exchange of information, goods, services, and payments associated with market transactions; and iii) providing an institutional infrastructure, such as legal and regulatory frameworks. The emergence of electronic marketplaces changed several of the business processes, which resulted in economic efficiencies. Also, the process of doing business in the virtual world is changing. Instead of handling raw materials, e-commerce involves gathering, selecting, synthesizing, and distributing of information.

Business models: The literature about e-commerce is not consistent in the usage of the term business model. A business model [8] can be defined as the architecture for the flow of products, services, and information, including a description of the various business actors and their roles.

The most common business models are e-shops, eprocurements, e-auctions, e-malls, third party marketplaces, virtual communities, value chain service providers, value chain integrators, collaboration platforms, and information brokerage to mention a few. Business models can be either electronic implementations of traditional forms of doing business, a complete integration of the whole value chain into the e-business environment, or some scalable degree in between.

Actors: The major actors of e-commerce can be categorized as buyers, sellers, intermediaries, and support services.

Figure 2 shows the relationships between the threedimensional model of e-business transactions in the context of the three key issues of added business value, interoperability, and trustworthiness.

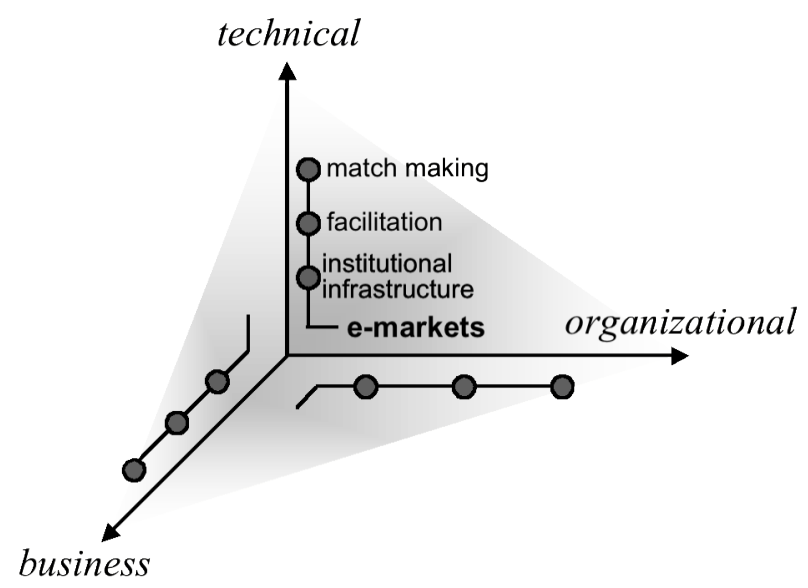

Figure 2. Relationships within the framework

\section{The Roles of Agents as Mediators in Electronic Commerce}

E-commerce applications can have many different forms [4]. In general they all follow the following pattern: need identification, information exchange on products and companies, negotiation/price discovery, contract establishment, contract fulfillment, and guarantee and feedback. 
This pattern encompasses three processes. First, the information about markets, participants and business models. This includes information on the available products or services, their respective specifications, suppliers, and delivery terms, as well as market mechanisms. Second, the negotiation process, which is a kind of decision-making process where involved parties in the e-business transaction jointly reach common decisions or solutions according to business models and market mechanisms. Third, the exchange process completing the transaction by exchanging goods and services for payments.

With the tremendous variety of e-commerce applications, the number of buyers, sellers, and transactions is growing rapidly [6]. In order for e-commerce to fully leverage business processes and to support efficiency and reliability, e-commerce transactions should be automated. However, e-commerce nowadays still requires some level of human intervention. The achievable level of automation depends on the type of the market, business model, and the business actors participating in the transaction.

In the following section, we elaborate on the roles that agents can play in the integral value added chain of ecommerce in the context of the markets - actors - business models framework developed in the previous section.

\subsection{Agents}

The term "agents" is used in such diversity that it has become meaningless without reference to a particular application. A commonly found definition of agents [11] is that they are software entities, which have some degree of autonomy, function continuously in an environment where other processes take place and other agents exist, carry out operations on behalf of a user or other programs, represent or have knowledge of the user's goals. This implies that agents should be autonomous, pro-active, reactive, and have sociability.

Autonomy [10] means that agents operate without direct intervention of humans or other agents, and have control over their actions and internal state. Pro-activeness means that agents do not simply act in response to their environment, but they are able to exhibit goal-oriented behavior by taking the initiative. Reactivity means that agents perceive their environment including the physical world, users, and other agents, and respond in timely fashion to changes observed. Sociability means that agents interact with other agents via some kind of communication language.

There are of course other interesting properties of agents such as mobility and learning [2]. Mobility indicates that agents might move from one machine to another. Learning provides agents with the ability to adopt their behavior on the basis of their experience.
Existing agents do not offer the majority of properties sketched above. However, the roles of agents in ecommerce may be well defined by these properties.

The first task within business transactions that agents could support is making contacts, establishing connections, negotiating and facilitating interactions among business parties [6]. They can disseminate information to a wide audience or automatically target information to those with special interests.

Collecting and filtering information constitutes the second task that agents could support. Agents can move over networks to gather information based on ad-hoc or pre-defined needs, compare it, and filter the best information based on the past experience of the user [4]. Moreover, the third and penultimate type of task that agents are good at is to provide a common interface for the entire business value added chain. To achieve this, agents can be configured with appropriate knowledge bases, task specific information, negotiation and communication protocols [1]. Finally, the forth task agents can perform well is to guide and monitor long-term complex processes that need frequent attention and have a life span of several hours to several months.

In the context of the agent-based model developed in the previous section, three different roles for agents can be identified: i) to support one or different parties in the trading process, ii) to provide the institutional infrastructure for electronic markets, and iii) to identify the possible ways for integrating functionality of different business models.

Figure 3 shows the different roles of agents in the context of the model developed earlier.

Agents as delegates: E-business actors can use agents in two different ways. First, agents can crawl the Internet to find the best bargain. Second, agents can buy or sell on behalf of their owners. In both cases, agents can help the user to decide which products to buy or sell, finding specifications and reviews, making recommendations, comparing different products based on a specific criteria, etc. This kind of agents can interact with each other and are even allowed to take part in the procurement activities such as request for goods, bid to supply the required goods, evaluate bids, place orders, and after the receipt of goods make payments.

Agents in markets: There seem to be at least two different cases where agents are able to provide the institutional infrastructure required for electronic markets [2] in which aggregate demand meets aggregate supply. The first is that of cooperation where agents can help users to allocate sources, skills, and products. The second is that of coordination where agents can be used to set up the trading rules. These rules impose explicit objective requirements to the admission of goods and traders into the marketplace, and constrain the actors' behavior to only those actions that are necessary and sufficient for trading under these conditions. 


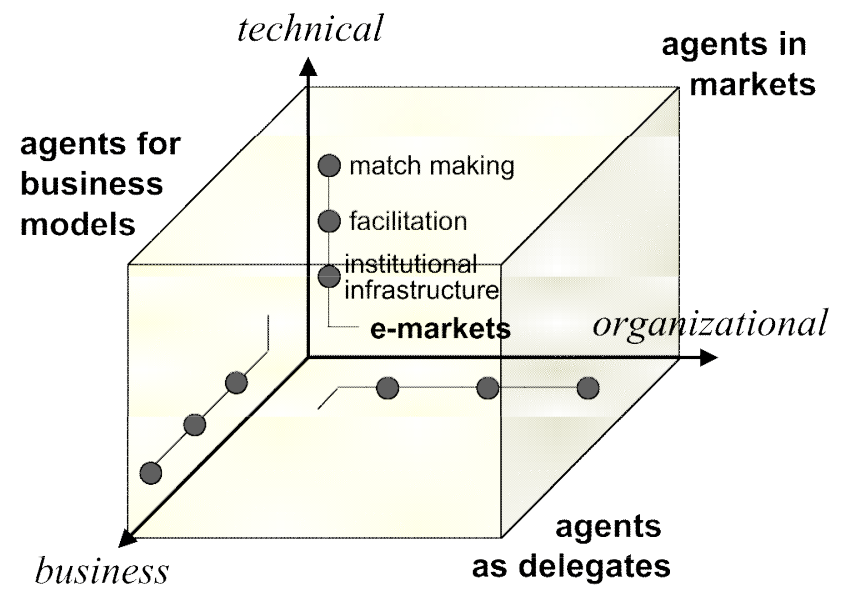

Figure 3. The role of agents as mediators

Agents for business models: Business models determine the way e-business transactions are conducted [4], and the required infrastructure to do them. They give a predefined structure for specifying the product and also facilitate the negotiation process (about the product's price).

For example, in the case of indirect markets there exists a business model in which a broker acts as mediator of the transaction. In case of an auction, the auction house performs this role. An auction house determines the procedure through which products can be delivered at the auction as well as an auction mechanism that determines the way products can be bought.

A rather new and very interesting approach is to design trading agents that function as intermediaries for the trading procedure. They have specialized knowledge on strategies and trading protocols to define the most suitable business model and to decide which trading procedure to follow.

\section{Conclusions and Future Work}

There is growing interest in the use of agents in ecommerce (for a survey, see [5]). Agents can be used in different settings where they offer support to users in locating appropriate goods and services, mediating ebusiness transactions, and reducing the information overheads.

There are currently many agent systems in the electronic commerce area, which perform limited functions to support users. Examples include Andersons Consulting's BargainFinder (http://bf.cstar.ac.com/bf), which undertakes price comparison, as does Jango (http://www.jango.com), AuctionBot (http://auction.eecs.umich.edu), and Kasbah (http://kasbah.media.mit.edu) - agent systems that support product transactions [6].

Agent mediated e-commerce is just developing its scientific and theoretical foundations. It requires a multidisciplinary approach to understand the different issues involved in the successful application of agents in ecommerce. In this paper we have presented a model for agents as mediators in e-commerce, which highlights how far technology can be used to automate e-business transactions.

Future work will concentrate on the systematic study of the roles of agents in the integral value added chain of ecommerce.

\section{References}

[1] Dignum, F., "Agent Communication and Cooperative Information Agents", in M. Klusch and L. Kerschberg (eds.) Cooperative Information Agents IV - The Future of Information Agents in Cyberspace (LNCS-1860), SpringerVerlag, 2000, pp. 191-207.

[2] Dignum, F., and Cortés, U., Agent-Mediated Electronic Commerce III (LNCS-2003), Springer-Verlag, 2001.

[3] Dignum, F., and Greaves, M., Issues in Agent Communication (LNCS-1916), Springer-Verlag, 2000.

[4] Dignum, F., Sierra, C., Agent Mediated Electronic Commerce. The European AgentLink Perspective (LNCS1993), Springer-Verlag, 2001.

[5] Guttman, R. H., Moukas, A. G., and Maes, P., "Agents as Mediators in Electronic Commerce", Electronic Markets, Vol. 8, No. 1, 1998, pp. 22-27.

[6] Macredie, R. D., "Mediating Buyer-Seller Interactions: The Role of Agents in Web Commerce", Electronic Markets, Vol. 8, No. 3, 1998, pp. 40-43.

[7] Schmid, B. F., "Electronic Markets", Electronic Markets, No. 9-10, 1993, pp. 3-4.

[8] Timmers, P., "Business Models for Electronic Markets", Electronic Markets, Vol. 8, No. 2, 1998, pp. 3-8.

[9] Turban, E., Lee, J., King, D., and Chung, H. M., Electronic Commerce: A managerial perspective, Prentice-Hall, Inc. NJ. 2000 .

[10] Verharen, E. "A Language-Action perspective on the design of cooperative information agents", Ph.D. thesis, Katholieke Universiteit Brabant, Tilburg, 1997.

[11] Wooldridge, M. "Intelligent Agents", in G. Weiss (ed.) Multiagent systems: A modern approach to distributed artificial intelligence, MIT Press, 1999, pp. 27-78. 